This is a postprint version of the following published document:

Marcellán, F., \& Varma, S. (2014). On an inverse problem for a linear combination of orthogonal polynomials. Journal of Difference Equations and Applications, 20, pp. 570-585.

DOI: $\underline{10.1080 / 10236198.2013 .86} \underline{4287}$

(C) Taylor \& Francis, 2014 


\title{
On an inverse problem for a linear combination of orthogonal polynomials
}

\author{
Francisco Marcellán ${ }^{\mathrm{a} *}$ and Serhan Varma ${ }^{\mathrm{b} 1}$ \\ ${ }^{a}$ Departamento de Matemáticas, Universidad Carlos III de Madrid, Avenida de la Universidad 30, \\ 28911 Leganés, Spain; ${ }^{b}$ Department of Mathematics, Faculty of Science, Ankara University, \\ Tandogan TR 06100, Ankara, Turkey
}

(Received 15 May 2013; accepted 5 November 2013)

This paper deals with the analysis of the orthogonality of a monic polynomial sequence $\left\{Q_{n}\right\}_{n \geq 0}$ defined as a linear combination of a sequence of monic orthogonal polynomials $\left\{P_{n}\right\}_{n \geq 0}$ with

$$
Q_{n}(x)=P_{n}(x)+s_{n} P_{n} \quad{ }_{1}(x)+t_{n} P_{n} \quad 2(x)+r_{n} P_{n} \quad 3(x), \quad n \geq 0,
$$

where $r_{n} \neq 0$ for $n \geq 3$. Moreover, we obtain the relation between the corresponding linear functionals as well as an explicit expression for the sequence of monic orthogonal polynomials $\left\{Q_{n}\right\}_{n \geq 0}$. We obtain the connection between the Jacobi matrices associated with $\left\{P_{n}\right\}_{n \geq 0}$ and $\left\{Q_{n}\right\}_{n \geq 0}$, respectively, by using an LU factorization. Some special cases of the above type relation are analysed.

Keywords: orthogonal polynomials; linear functionals; Jacobi matrices; inverse problems

MSC (2010) Classification: 33C45; 42C05

\section{Introduction}

Let $\mathcal{P}$ be the linear space of polynomials with complex coefficients and $\mathcal{P}^{\prime}$ be its algebraic dual. We denote by $\langle u, f\rangle$ the action of the linear functional $u \in \mathcal{P}^{\prime}$ on the polynomial $f \in \mathcal{P}$. A sequence of monic polynomials $\left\{P_{n}\right\}_{n \geq 0}$ is called orthogonal with respect to the linear functional $u$ if the following orthogonality conditions hold

$$
\begin{cases}\left\langle u, P_{n} P_{m}\right\rangle \quad 0, & n \neq m, \\ \left\langle u, P_{n}^{2}\right\rangle \neq 0, & n \quad 0,1, \ldots,\end{cases}
$$

where $\operatorname{deg} P_{n} \quad n$ for every $n \quad 0,1, \ldots$ (see [6]).

The linear functional $u$ is called quasi-definite (regular) if the leading principal submatrices $H_{n}$ of the Hankel matrix $H \quad\left(u_{i+j}\right)_{i, j \geq 0}$ related to the moments $u_{k} \quad\left\langle u, x^{k}\right\rangle$, $k \geq 0$, are non-singular for each $n \geq 0$ (see [6]).

The following theorem gives a well-known characterization for the sequence of monic orthogonal polynomials (SMOP) $\left\{P_{n}\right\}_{n \geq 0}$ associated with the quasi-definite linear functional $u$.

Corresponding author. Email: pacomarc@ing.uc3m.es 
THEOREM 1.1. Let u be a quasi-definite linear functional and $\left\{P_{n}\right\}_{n \geq 0}$ be the corresponding SMOP. Then, $\left\{P_{n}\right\}_{n \geq 0}$ satisfies the following three-term recurrence relation

$$
P_{n+1}(x) \quad\left(\begin{array}{ll}
x & \beta_{n}
\end{array}\right) P_{n}(x) \quad \gamma_{n} P_{n}{ }_{1}(x), \quad n \geq 0, \quad P_{1}(x) \quad 0, \quad P_{0}(x) \quad 1,
$$

where $\left\{\beta_{n}\right\}_{n \geq 0}$ and $\left\{\gamma_{n}\right\}_{n \geq 1}$ are sequences of complex numbers with $\gamma_{n} \neq 0$ for each $n \geq 1$.

Conversely, if a sequence of monic polynomials $\left\{P_{n}\right\}_{n \geq 0}$ satisfies a three-term recurrence relation like (1.1), then there exists a unique quasi-definite linear functional $u$ such that $\left\{P_{n}\right\}_{n \geq 0}$ is the corresponding SMOP. This result is known in the literature as Favard's Theorem (see [6]).

Let $\left\{P_{n}^{(1)}\right\}_{n \geq 0}$ be the associated SMOP of the first kind related to an SMOP $\left\{P_{n}\right\}_{n \geq 0}$ with respect to $u$. Then, $\left\{P_{n}\right\}_{n \geq 0}$ is defined by the three-term recurrence relation

$$
P_{n+1}^{(1)}(x) \quad\left(x \quad \beta_{n+1}\right) P_{n}^{(1)}(x) \quad \gamma_{n+1} P_{n}^{(1)}(x), \quad n \geq 0, \quad P_{1}^{(1)}(x) \quad 0, \quad P_{0}^{(1)}(x) \quad 1 .
$$

Furthermore, $P_{n}^{(1)}(x) \quad\left(1 / u_{0}\right)\left\langle u,\left(P_{n+1}(x) \quad P_{n+1}(t)\right) /(x \quad t)\right\rangle$ where the linear functional $u$ acts on the variable $t$ (see [6]). Also, we will denote by $\left\{P_{n}(., \alpha)\right\}_{n \geq 0}$ the co-recursive SMOP defined by

$$
\begin{array}{rllllll}
P_{n+1}(x, \alpha) & (x & \left.\beta_{n}\right) P_{n}(x, \alpha) & \gamma_{n} P_{n}(x, \alpha), \quad n \geq 1, \quad P_{1}(x, \alpha) \quad P_{1}(x) \quad \alpha, \\
P_{0}(x, \alpha) & 1 . &
\end{array}
$$

The following connection formula is widely known in the literature (see [7])

$$
P_{n}(x, \alpha) \quad P_{n}(x) \quad \alpha P_{n}^{(1)}(x), \quad n \geq 1 .
$$

In the theory of orthogonal polynomials, the well-known basic canonical spectral transformations of a quasi-definite linear functional $u$ are (see [24])

(i) Christoffel transformation

$$
v_{1} \quad(x \quad a) u,
$$

(ii) Uvarov transformation

$$
v_{2} \quad u+M \delta_{a},
$$

(iii) Geronimus transformation

$$
v_{3} \quad(x \quad a){ }^{1} u+M \delta_{a},
$$

where the left multiplication of a linear functional $u \in \mathcal{P}^{\prime}$ by a polynomial $f \in \mathcal{P}$ is defined by

$$
\langle f u, p\rangle \quad\langle u, f p\rangle, \quad p \in \mathcal{P},
$$

division of $u$ by a first-degree polynomial

$$
\left\langle\left(\begin{array}{ll}
x & a
\end{array}{ }^{1} u, p\right\rangle \quad\left\langle u, \frac{p(x) \quad p(a)}{x \quad a}\right\rangle, \quad p \in \mathcal{P} .\right.
$$


There, $\delta_{a}$ is the Dirac functional at the mass point $a$ given by

$$
\left\langle\delta_{a}, p\right\rangle \quad p(a), \quad p \in \mathcal{P},
$$

and $M \in \mathbb{R}$.

Necessary and sufficient conditions under which the linear functionals $v_{1}, v_{2}$ and $v_{3}$ are quasi-definite and the algebraic relations between the corresponding SMOPs are deeply investigated in the literature (see $[7,19,20,23,24]$ ).

Let $u$ be a quasi-definite linear functional and $\left\{P_{n}\right\}_{n \geq 0}$ be the corresponding SMOP. When there is an algebraic relation between $\left\{P_{n}\right\}_{n \geq 0}$ and a given monic polynomial sequence $\left\{Q_{n}\right\}_{n \geq 0}$ as

$$
Q_{n}(x)+\sum_{i=1}^{m} a_{i, n} Q_{n}{ }_{i}(x) \quad P_{n}(x)+\sum_{i=1}^{k} b_{i, n} P_{n} \quad(x), \quad n \geq 0,
$$

to find necessary and sufficient conditions such that $\left\{Q_{n}\right\}_{n \geq 0}$ is also orthogonal and the relation between the corresponding quasi-definite linear functionals is said to be an inverse problem. Here $m, k \in \mathbb{Z}^{+}$and $\left(a_{i, n}\right)_{n \geq 0}$ and $\left(b_{i, n}\right)_{n \geq 0}$ are sequences of complex numbers. The cases $m \quad 1, k \quad 2$ and $m \quad 2, k \quad 1$ have been studied in [16]. For the case $m \quad 2$, $k \quad 2$, one can find a detailed investigation in [3]. Later on, the situation $m \quad 1, k \quad N$, with constant coefficients has been considered in [4]. In this contribution, necessary and sufficient conditions in order for $\left\{Q_{n}\right\}_{n \geq 0}$ to be an SMOP are obtained, and a matrix method by using Jacobi matrices associated with $\left\{P_{n}\right\}_{n \geq 0}$ and $\left\{Q_{n}\right\}_{n \geq 0}$ is derived. Moreover, for the case $N \quad 3$, the authors described the recurrence coefficients $\left\{\beta_{n}\right\}_{n \geq 0}$ and $\left\{\gamma_{n}\right\}_{n \geq 1}$ corresponding to $\left\{P_{n}\right\}_{n \geq 0}$ such that $\left\{Q_{n}\right\}_{n \geq 0}$ is also orthogonal. Then, in [2] the authors dealt with the case $m \quad 1, k \quad 3$. The characterization of the orthogonality of sequence $\left\{Q_{n}\right\}_{n \geq 0}$, the relation between the quasi-definite linear functionals such that $\left\{P_{n}\right\}_{n \geq 0}$ and $\left\{Q_{n}\right\}_{n \geq 0}$ are the corresponding SMOPs and a matrix interpretation, where Jacobi matrices and Christoffel formula [10] play central role, are remarkably deduced. First time, this type of inverse problem was discussed in [5]. Recently, similar analysis has been done in [1] for the case $m \quad 2, k \quad 3$. On the other hand, the general linear structure relations given by (1.2) have been analysed in [22] with an additional assumption about the orthogonality of $\left\{Q_{n}\right\}_{n \geq 0}$.

In this paper, our aim was to focus our attention on the special case of the general linear structure relations (1.2) with $m \quad 1, k \quad$ 4, i.e. for $n \geq 0$

$$
Q_{n}(x) \quad P_{n}(x)+s_{n} P_{n} \quad 1(x)+t_{n} P_{n} \quad 2(x)+r_{n} P_{n} \quad 3(x),
$$

where $s_{n}, t_{n}$ and $r_{n}$ are complex numbers with the initial conditions $s_{0} \quad t_{0} \quad t_{1}$

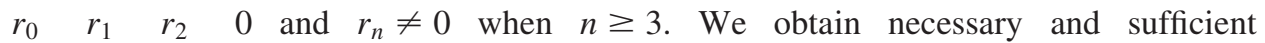
conditions for the orthogonality of the sequence of monic polynomials $\left\{Q_{n}\right\}_{n \geq 0}$. In addition, we get the relation between the linear functionals $u$ and $v$, respectively, corresponding to the SMOPs $\left\{P_{n}\right\}_{n \geq 0}$ and $\left\{Q_{n}\right\}_{n \geq 0}$ by $q(x) v \quad k u$, where $q(x)$ $x^{3}+a x^{2}+b x+c$ is a monic cubic polynomial and $k \in \mathbb{C} \backslash\{0\}$. It is worthy to note that for the special case $q(x) \quad x^{3}$, this problem was solved in [17]. Moreover, an explicit expression of the SMOP $\left\{Q_{n}\right\}_{n \geq 0}$ is derived. A connection between the corresponding monic Jacobi matrices is presented, and some special cases of relation (1.3) are finally discussed. 


\section{Basic results}

In this section, $\left\{P_{n}\right\}_{n \geq 0}$ will denote an SMOP with respect to a quasi-definite linear functional $u$. An immediate consequence for $\left\{P_{n}\right\}_{n \geq 0}$ is to satisfy the three-term recurrence relation (1.1) with recurrence coefficients $\left\{\beta_{n}\right\}_{n \geq 0}$ and $\left\{\gamma_{n}\right\}_{n \geq 1}$. First of all, we characterize the orthogonality of the sequence of monic polynomials $\left\{Q_{n}\right\}_{n \geq 0}$, which is related to $\left\{P_{n}\right\}_{n \geq 0}$ by (1.3).

THEOREM 2.1. Let $\left\{P_{n}\right\}_{n \geq 0}$ be a given SMOP and let $\left\{Q_{n}\right\}_{n \geq 0}$ be a sequence of polynomials given by (1.3), with $r_{n} \neq 0$ for all $n \geq 3$. Then $\left\{Q_{n}\right\}_{n \geq 0}$ is an SMOP with recurrence coefficients $\left\{\tilde{\beta}_{n}\right\}_{n \geq 0}$ and $\left\{\tilde{\gamma}_{n}\right\}_{n \geq 1}$ given by (2.5) and (2.6) if and only if the following conditions hold

$$
\tilde{\gamma}_{n} \quad \gamma_{n}+t_{n} \quad t_{n+1}+s_{n}\left(\beta_{n} 1 \quad \beta_{n} \quad s_{n}+s_{n+1}\right) \neq 0, \quad n \geq 1,
$$

as well as

$$
\begin{aligned}
& \begin{array}{lllllll}
s_{n} & 1 & \tilde{\gamma}_{n} & s_{n} \gamma_{n} & 1
\end{array} r_{n} \quad r_{n+1}+t_{n}\left(\begin{array}{llll}
\beta_{n} & 2 & \beta_{n} & s_{n}+s_{n+1}
\end{array}\right), \quad n \geq 2, \\
& \begin{array}{llllll}
t_{n} & 1 & \tilde{\gamma}_{n} & t_{n} \gamma_{n} & 2
\end{array}+r_{n}\left(\begin{array}{llll}
\beta_{n} & 3 & \beta_{n} & s_{n}+s_{n+1}
\end{array}\right), \quad n \geq 3, \\
& \begin{array}{llll}
r_{n} & 1 & \tilde{\gamma}_{n} & r_{n} \gamma_{n} 3
\end{array}, \quad n \geq 4,
\end{aligned}
$$

together with $\tilde{\gamma}_{1} \tilde{\gamma}_{2} \tilde{\gamma}_{3} \neq 0$. Furthermore, the coefficients of the three-term recurrence relation for $\left\{Q_{n}\right\}_{n \geq 0}$ are

$$
\begin{gathered}
\tilde{\beta}_{n} \quad \beta_{n}+s_{n} \quad s_{n+1}, \quad n \geq 0, \\
\tilde{\gamma}_{n} \quad \gamma_{n}+t_{n} \quad t_{n+1}+s_{n}\left(\begin{array}{llll}
\beta_{n} & 1 & \beta_{n} & \left.s_{n}+s_{n+1}\right), \quad n \geq 1 .
\end{array}\right.
\end{gathered}
$$

Proof. Multiplying each side of relation (1.3) by $x$, we obtain

$$
x Q_{n}(x) \quad x P_{n}(x)+s_{n} x P_{n} 1(x)+t_{n} x P_{n} 2(x)+r_{n} x P_{n} \quad 3(x), \quad n \geq 0 .
$$

Substituting recurrence relation (1.1) into (2.7) for $x P_{n}(x), x P_{n}{ }_{1}(x), x P_{n}{ }_{2}(x)$, and $x P_{n} 3(x)$, then using relation (1.3) for $P_{n+1}(x)$, we get for $n \geq 0$

$$
\begin{aligned}
& x Q_{n}(x) \quad Q_{n+1}(x)+\left(\beta_{n}+s_{n} \quad s_{n+1}\right) P_{n}(x) \\
& +\left[\begin{array}{lll}
\gamma_{n}+t_{n} & t_{n+1}+s_{n} \beta_{n} & 1
\end{array}\right] P_{n} \quad 1(x)
\end{aligned}
$$

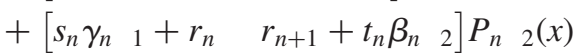

$$
\begin{aligned}
& +\left[\begin{array}{llll}
t_{n} \gamma_{n} & 2 & +r_{n} \beta_{n} & 3
\end{array}\right] P_{n}{ }_{3}(x)+r_{n} \gamma_{n}{ }_{3} P_{n}{ }_{4}(x)
\end{aligned}
$$

with the convention $P_{n}(x) \quad 0, n \geq 1$. By using relation (1.3) for $P_{n}(x)$, the last equality becomes for $n \geq 0$

$$
\begin{aligned}
x Q_{n}(x) & Q_{n+1}(x)+\tilde{\beta}_{n} Q_{n}(x) \\
& +\left[\begin{array}{llllll}
\gamma_{n}+t_{n} & t_{n+1}+s_{n}\left(\beta_{n}\right. & 1 & \beta_{n} & \left.s_{n}+s_{n+1}\right)
\end{array}\right] P_{n} 1_{1}(x) \\
& +\left[\begin{array}{llllll}
s_{n} \gamma_{n} & 1+r_{n} & r_{n+1}+t_{n}\left(\beta_{n}\right. & 2 & \beta_{n} & \left.s_{n}+s_{n+1}\right)
\end{array}\right] P_{n} 2_{2}(x) \\
& +\left[\begin{array}{llllll}
t_{n} \gamma_{n} & 2+r_{n}\left(\begin{array}{lllll}
\beta_{n} & 3 & \beta_{n} & \left.s_{n}+s_{n+1}\right)
\end{array}\right] P_{n} & 3 \\
3 & (x)+r_{n} \gamma_{n} & { }_{3} P_{n} & 4
\end{array}(x),\right.
\end{aligned}
$$


where $\tilde{\beta}_{n}$ is exactly given by (2.5). Then, according to relation (1.3) for $P_{n}{ }_{1}(x)$, we have for $n \geq 0$

$$
\begin{aligned}
& x Q_{n}(x) \quad Q_{n+1}(x)+\tilde{\beta}_{n} Q_{n}(x)+\tilde{\gamma}_{n} Q_{n} \quad 1(x)
\end{aligned}
$$

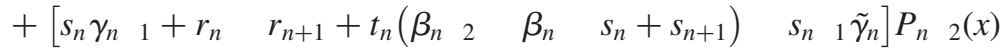

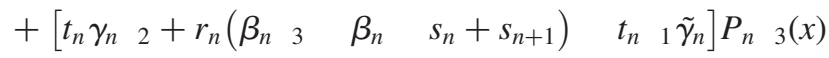

$$
\begin{aligned}
& +\left[\begin{array}{llll}
r_{n} \gamma_{n} & 3 & r_{n} & 1 \\
\gamma_{n}
\end{array}\right] P_{n}{ }_{4}(x),
\end{aligned}
$$

where $\tilde{\gamma}_{n}$ is given by (2.6). Thus, from (2.8), $\left\{Q_{n}\right\}_{n \geq 0}$ is an SMOP if and only if conditions (2.1) (2.4) are satisfied.

Remark 2.2. Taking into account Theorem 2.1, given an SMOP $\left\{P_{n}\right\}_{n \geq 0}$ in order for $\left\{Q_{n}\right\}_{n \geq 0}$ to be an SMOP, the coefficients $s_{n+1}, t_{n+1}$, and $r_{n+1}$ can be generated from the previous coefficients as follows from (2.3) and (2.4):

$$
s_{n+1} \quad s_{n}+\beta_{n} \quad \beta_{n} 3+\frac{t_{n} 1}{r_{n} 1} \gamma_{n} 3 \quad \frac{t_{n}}{r_{n}} \gamma_{n 2},
$$

from (2.6) and (2.4):

$$
t_{n+1} \quad t_{n}+s_{n}\left(\begin{array}{llll}
\beta_{n} & 1 & \beta_{n} & 3
\end{array}\right)+\gamma_{n}+s_{n}\left(\begin{array}{llll}
\frac{t_{n}}{r_{n}} \gamma_{n} & 3 & \frac{t_{n}}{r_{n}} \gamma_{n} & 2
\end{array}\right) \quad \frac{r_{n}}{r_{n} \gamma_{n}} \gamma_{n} 3,
$$

and from (2.2) and (2.4):

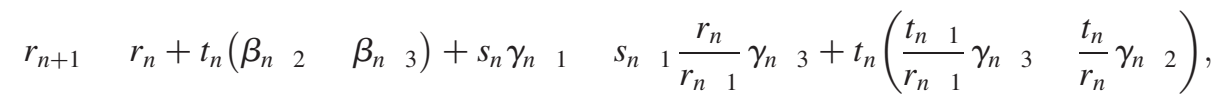

for $n \geq 4$. Note that $s_{1}, s_{2}, s_{3}, t_{2}, t_{3}$ and $r_{3}$ are free parameters.

On the other hand, the first previous relation (equivalent to (2.1)) can be written as

$$
s_{n+1} \quad \beta_{n} \quad \beta_{n} 1 \quad \beta_{n} 2+\frac{t_{n}}{r_{n}} \gamma_{n} 2 s_{n} \quad \beta_{n} 1 \quad \beta_{n} 2 \quad \beta_{n} 3+\frac{t_{n} 1}{r_{n} \gamma_{n}} \gamma_{n}, \quad n \geq 4,
$$

and so there exists a constant $A$ such that

$$
s_{n+1} \quad \beta_{n} \quad \beta_{n} 1 \quad \beta_{n} 2+\frac{t_{n}}{r_{n}} \gamma_{n} 2 \quad A, \quad n \geq 3,
$$

Next, we state that there is a relation between quasi-definite linear functionals when $\left\{Q_{n}\right\}_{n \geq 0}$ is an SMOP with respect to a quasi-definite linear functional $v$. If the sequence of linear functionals $\left\{\omega_{n}\right\}_{n \geq 0}$ according to the SMOP $\left\{P_{n}\right\}_{n \geq 0}$ satisfies the condition $\left\langle\omega_{n}, P_{m}\right\rangle \quad \delta_{n m}$, then $\left\{\omega_{n}\right\}_{n \geq 0}$ is called the dual basis of $\left\{P_{n}\right\}_{n \geq 0}$ where $\delta_{n m}$ is the Kronecker delta. It is well known that

$$
\omega_{n} \frac{P_{n} u}{\left\langle u, P_{n}^{2}\right\rangle} .
$$

THEOREM 2.3. (see [18]) Let $\left\{P_{n}\right\}_{n \geq 0}$ be an SMOP with respect to a quasi-definite linear functional $u$ and the sequence of monic polynomials $\left\{Q_{n}\right\}_{n \geq 0}$ be given by relation (1.3). If $\left\{Q_{n}\right\}_{n \geq 0}$ is an SMOP with respect to a quasi-definite linear functional $v$, then

$$
q(x) v \quad k u
$$


where $q(x) \quad x^{3}+a x^{2}+b x+c, k \in \mathbb{C} \backslash\{0\}$ and the normalizations for these linear functionals $\langle u, 1\rangle \quad\langle v, 1\rangle \quad 1$.

Proof. Applying the quasi-definite linear functional $u$ corresponding to the SMOP $\left\{P_{n}\right\}_{n \geq 0}$ in (1.3), we get for $n \geq 4$

$$
\left\langle u, Q_{n}\right\rangle \quad 0 .
$$

Then, according to [20] and taking into account (1.3), we expand the linear functional $u$ in terms of the dual basis $\left\{\left(Q_{j} v\right) /\left\langle v, Q_{j}^{2}\right\rangle\right\}_{j \geq 0}$ of the SMOP $\left\{Q_{n}\right\}_{n \geq 0}$ as

$$
u \quad \sum_{j=0}^{3} \frac{\left\langle u, Q_{j}\right\rangle}{\left\langle v, Q_{j}^{2}\right\rangle} Q_{j} v \quad\left[1+\frac{s_{1}}{\left\langle v, Q_{1}^{2}\right\rangle} Q_{1}+\frac{t_{2}}{\left\langle v, Q_{2}^{2}\right\rangle} Q_{2}+\frac{r_{3}}{\left\langle v, Q_{3}^{2}\right\rangle} Q_{3}\right] v
$$

where $\left\langle u, Q_{0}\right\rangle \quad 1,\left\langle u, Q_{1}\right\rangle \quad s_{1},\left\langle u, Q_{2}\right\rangle \quad t_{2}$ and $\left\langle u, Q_{3}\right\rangle \quad r_{3}$.

Since $\left\{Q_{n}\right\}_{n \geq 0}$ is the SMOP with respect to $v$, the recurrence coefficients $\left\{\tilde{\beta}_{n}\right\}_{n \geq 0}$ and $\left\{\tilde{\gamma}_{n}\right\}_{n \geq 1}$ are given as in (2.5) and (2.6). Furthermore,

$$
\tilde{\gamma}_{n} \frac{\left\langle v, Q_{n}^{2}\right\rangle}{\left\langle v, Q_{n}^{2} 1\right\rangle} \neq 0, \quad n \geq 1
$$

Combining (2.10) and relation (1.3) with (2.9), we get the desired result with $k$ $\left(\tilde{\gamma}_{1} \tilde{\gamma}_{2} \tilde{\gamma}_{3}\right) / r_{3}$ where $\tilde{\gamma}_{1}, \tilde{\gamma}_{2}$ and $\tilde{\gamma}_{3}$ are given by $(2.6)$.

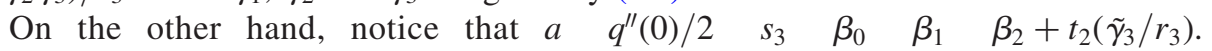
According to relation (2.3), we get $a \quad s_{4} \quad \beta_{1} \quad \beta_{2} \quad \beta_{3}+t_{3}\left(\gamma_{1} / r_{3}\right) \quad A$.

Now, we will find the explicit expression of the SMOP $\left\{Q_{n}\right\}_{n \geq 0}$ given by relation (1.3).

THEOREM 2.4. Let $\left\{P_{n}\right\}_{n \geq 0}$ be an SMOP with respect to a quasi-definite linear functional $u$, $\left\{Q_{n}\right\}_{n \geq 0}$ be given by relation (1.3) and let us assume that the cubic polynomial $q(x)$ has simple zeros $\alpha_{1}, \alpha_{2}, \alpha_{3}$. If $\left\{Q_{n}\right\}_{n \geq 0}$ is an SMOP with respect to a quasi-definite linear functional $v$, then for $n \geq 3$

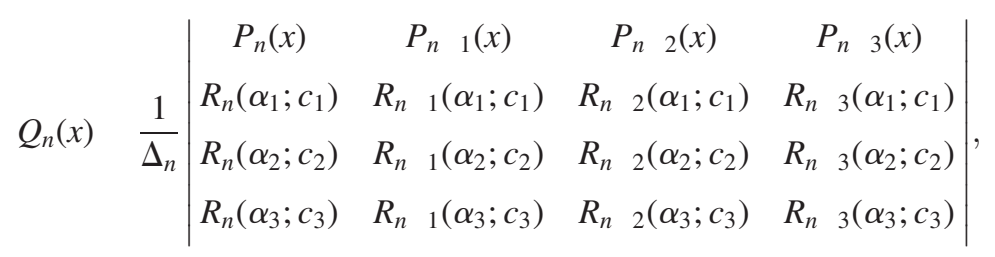

where

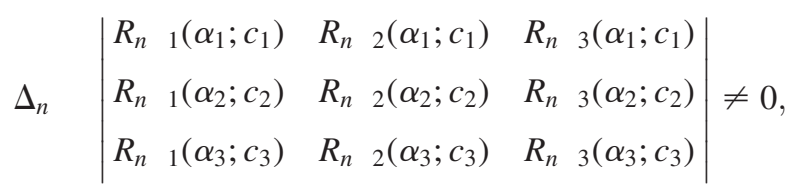


$R_{n}(x ; \eta) \quad \eta P_{n}(x)+P_{n}^{(1)}{ }_{1}(x), \eta \in \mathbb{C}, k \in \mathbb{C} \backslash\{0\},\langle u, 1\rangle \quad\langle v, 1\rangle \quad 1$, and

$$
\begin{aligned}
& c_{1} \frac{1}{k}\left\langle v,\left(x \quad \alpha_{2}\right)\left(x \quad \alpha_{3}\right)\right\rangle, \\
& c_{2} \frac{1}{k}\left\langle v,\left(x \quad \alpha_{1}\right)\left(x \quad \alpha_{3}\right)\right\rangle \text {, } \\
& c_{3} \frac{1}{k}\left\langle v,\left(x \quad \alpha_{1}\right)\left(x \quad \alpha_{2}\right)\right\rangle .
\end{aligned}
$$

Proof. Assume that $\left\{Q_{n}\right\}_{n \geq 0}$ is an SMOP with respect to $v$. Then, according to Theorem 2.3,

$$
q(x) v \quad k u .
$$

On one hand, if we take $x \quad \alpha_{1}$ at relation (1.3), then we obtain

$$
Q_{n}\left(\alpha_{1}\right) \quad P_{n}\left(\alpha_{1}\right)+s_{n} P_{n}{ }_{1}\left(\alpha_{1}\right)+t_{n} P_{n}{ }_{2}\left(\alpha_{1}\right)+r_{n} P_{n} \quad{ }_{3}\left(\alpha_{1}\right), \quad n \geq 3 .
$$

Substracting the last equality from relation (1.3), dividing each side by $x \quad \alpha_{1}$ and after applying the linear functional $u$, we get

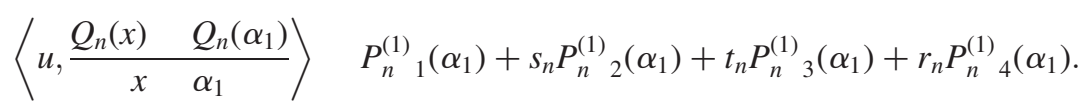

At the left-hand side of (2.12), we get by using (2.11) for $n \geq 3$

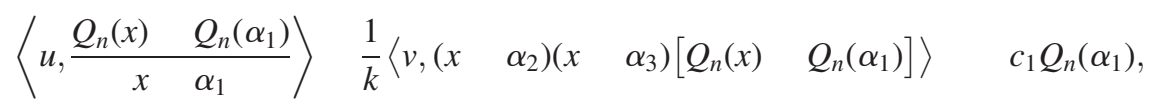

where

$$
c_{1} \quad \frac{1}{k}\left\langle v,\left(x \quad \alpha_{2}\right)\left(x \quad \alpha_{3}\right)\right\rangle .
$$

Thus, for $n \geq 3$

$$
c_{1} Q_{n}\left(\alpha_{1}\right) \quad P_{n}^{(1)}{ }_{1}\left(\alpha_{1}\right)+s_{n} P_{n}^{(1)}\left(\alpha_{1}\right)+t_{n} P_{n}^{(1)}{ }_{3}\left(\alpha_{1}\right)+r_{n} P_{n}^{(1)}{ }_{4}\left(\alpha_{1}\right) .
$$

Taking into account relation (1.3) and the definition of the polynomials $R_{n}(x ; \eta)$ in the last equality, we have for $n \geq 3$

$$
R_{n}\left(\alpha_{1} ; c_{1}\right) \quad s_{n} R_{n}{ }_{1}\left(\alpha_{1} ; c_{1}\right)+t_{n} R_{n}{ }_{2}\left(\alpha_{1} ; c_{1}\right)+r_{n} R_{n}{ }_{3}\left(\alpha_{1} ; c_{1}\right) .
$$

After similar computations, it follows for $n \geq 3$

$$
\begin{aligned}
& R_{n}\left(\alpha_{2} ; c_{2}\right) \quad s_{n} R_{n}{ }_{1}\left(\alpha_{2} ; c_{2}\right)+t_{n} R_{n}{ }_{2}\left(\alpha_{2} ; c_{2}\right)+r_{n} R_{n}{ }_{3}\left(\alpha_{2} ; c_{2}\right), \\
& R_{n}\left(\alpha_{3} ; c_{3}\right) \quad s_{n} R_{n}{ }_{1}\left(\alpha_{3} ; c_{3}\right)+t_{n} R_{n}{ }_{2}\left(\alpha_{3} ; c_{3}\right)+r_{n} R_{n}{ }_{3}\left(\alpha_{3} ; c_{3}\right),
\end{aligned}
$$

where

$$
c_{2} \frac{1}{k}\left\langle v,\left(x \quad \alpha_{1}\right)\left(x \quad \alpha_{3}\right)\right\rangle, \quad c_{3} \quad \frac{1}{k}\left\langle v,\left(x \quad \alpha_{1}\right)\left(x \quad \alpha_{2}\right)\right\rangle .
$$

Hence, we reach the desired result from (2.13) (2.15) and relation (1.3). 
Remark 2.5. Let $\alpha_{1}$ be a simple zero and $\alpha_{2}$ be a zero with multiplicity 2 of the cubic polynomial $q(x)$. Then, taking into account Theorem 2.4, substracting (2.15) from (2.14) and after dividing each side by $\alpha_{2} \quad \alpha_{3}$, when $\alpha_{3}$ tends to $\alpha_{2}$. We obtain

$$
R_{n}^{\prime}\left(\alpha_{2} ; c_{3}\right) \quad s_{n} R_{n}^{\prime}{ }_{1}\left(\alpha_{2} ; c_{3}\right)+t_{n} R_{n}^{\prime}{ }_{2}\left(\alpha_{2} ; c_{3}\right)+r_{n} R_{n}^{\prime}{ }_{3}\left(\alpha_{2} ; c_{3}\right),
$$

where we take $\beta_{0} \quad s_{1} \quad \alpha_{1}$ and $\left(\beta_{0}+\beta_{1} \quad s_{2}\right) / 2 \neq \alpha_{2}$. Notice that $k\langle v, q(x)\rangle \in \mathbb{C} \backslash\{0\}$. In a similar way, we get the explicit expression of the SMOP $\left\{Q_{n}\right\}_{n \geq 0}$ from (2.13), (2.14) and (2.16) by

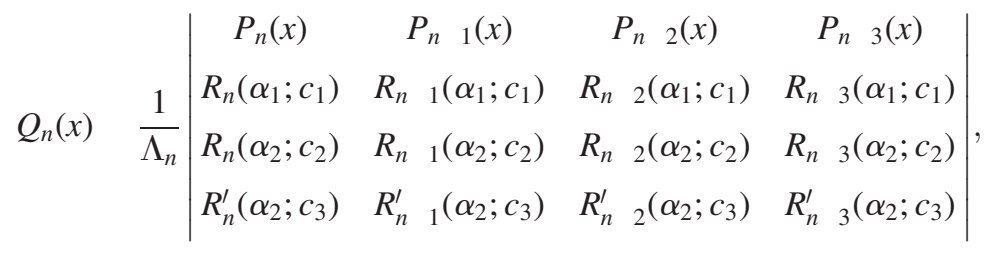

where $n \geq 3, \beta_{0} \quad s_{1} \quad \alpha_{1},\left(\beta_{0}+\beta_{1} \quad s_{2}\right) / 2 \neq \alpha_{2}$ and

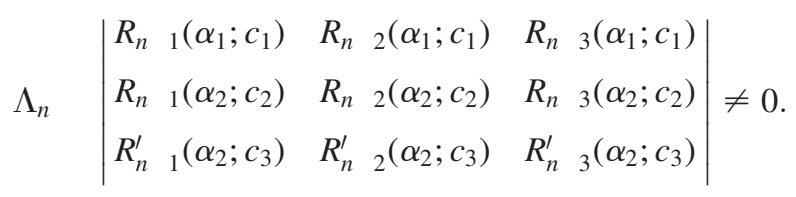

In this situation, notice that

$$
c_{1} \quad \frac{1}{k}\left\langle v,\left(x \quad \alpha_{2}\right)^{2}\right\rangle, \quad c_{2} \quad c_{3} \quad \frac{1}{k}\left\langle v,\left(x \quad \alpha_{1}\right)\left(x \quad \alpha_{2}\right)\right\rangle .
$$

In addition, if the cubic polynomial $q(x)$ has a zero with multiplicity 3 , then one can find a detailed study in [17].

\subsection{Jacobi matrices and inverse cubic polynomial transformations}

Let $\mathbf{P} \quad\left(P_{0}, P_{1}, \ldots\right)^{\mathrm{T}}$ and $\mathbf{Q} \quad\left(Q_{0}, Q_{1}, \ldots\right)^{\mathrm{T}}$ be the column vectors associated with the SMOPs $\left\{P_{n}\right\}_{n \geq 0}$ and $\left\{Q_{n}\right\}_{n \geq 0}$, respectively. From Theorem 1.1, $\left\{P_{n}\right\}_{n \geq 0}$ and $\left\{Q_{n}\right\}_{n \geq 0}$ satisfy the three-term recurrence relation of form (1.1) which can be read

$$
x \mathbf{P} \quad \mathbf{J}_{P} \mathbf{P}, \quad x \mathbf{Q} \quad \mathbf{J}_{Q} \mathbf{Q},
$$

where $\mathbf{J}_{P}$ and $\mathbf{J}_{Q}$ are the corresponding monic Jacobi matrices. Now, we explain a method in order to find the matrix $\mathbf{J}_{Q}$ with the help of the matrix $\mathbf{J}_{P}$, the polynomial $q(x)$ $x^{3}+a x^{2}+b x+c$ and relation (1.3). Taking into account relation (1.3), we have

$$
\text { Q AP, }
$$

where $\mathbf{A} \quad\left(a_{i, j}\right)_{i, j \geq 1}$ is a banded lower triangular matrix with $a_{i, i} \quad 1$ and $a_{i, j} \quad 0$ for $i \quad j>3$. Then, according to (2.17) and (2.18), we obtain

$$
x \mathbf{A P} \quad \mathbf{J}_{Q} \mathbf{A P}, \quad \text { i.e. } \quad \mathbf{A J}_{P} \quad \mathbf{J}_{Q} \mathbf{A}
$$


and, as a consequence,

$$
\mathbf{J}_{Q} \quad \mathbf{A} \mathbf{J}_{P} \mathbf{A}^{1} .
$$

Besides, it follows from Christoffel formula [10] that

$$
q(x) \mathbf{P} \quad \mathbf{B Q},
$$

where $\mathbf{B} \quad\left(b_{i, j}\right)_{i, j \geq 1}$ is a banded upper triangular matrix with $b_{i, i+3} \quad 1$ and $b_{i, j} \quad 0$ for $j \quad i>3$. Combining (2.17) and (2.18) with (2.20) provides

$$
q\left(\mathbf{J}_{P}\right) \quad \mathbf{B A} .
$$

Notice that $q\left(\mathbf{J}_{p}\right)$ is a seventh-diagonal matrix. Consequently, from equality (2.21), we can find the matrix B. On the other hand, in view of equalities (2.19) and (2.21), we get

$$
q\left(\mathbf{J}_{Q}\right) \quad \mathbf{A} q\left(\mathbf{J}_{P}\right) \mathbf{A}^{1} \quad \mathbf{A B} .
$$

Finally, we find the matrix $\mathbf{J}_{Q}$ from the last equality. Notice that we have extended the standard Geronimus transformation to a cubic case. Thus (2.21) is the UL factorization of the matrix $q\left(\mathbf{J}_{P}\right)$ and we have three free parameters that are the masses of the linear functional $v$ at the zeros of the polynomial $q(x)$.

Next, we describe a method to state the connection between the truncated monic Jacobi matrices. Indeed, for $(\mathbf{P})_{n} \quad\left(P_{0}, P_{1}, \ldots, P_{n}\right)^{\mathrm{T}}$ and $(\mathbf{Q})_{n} \quad\left(Q_{0}, Q_{1}, \ldots, Q_{n}\right)^{\mathrm{T}}$, the truncated form of three-term recurrence relations (2.17) and relation (2.18) is

$$
\begin{array}{cl}
x(\mathbf{P})_{n} & \left(\mathbf{J}_{P}\right)_{n+1}(\mathbf{P})_{n}+P_{n+1} e_{n+1} \\
x(\mathbf{Q})_{n} & \left(\mathbf{J}_{Q}\right)_{n+1}(\mathbf{Q})_{n}+Q_{n+1} e_{n+1}
\end{array}
$$

$$
(\mathbf{Q})_{n} \quad(\mathbf{A})_{n+1}(\mathbf{P})_{n},
$$

where the symbol $(.)_{n}$ denotes the truncation of any infinite matrix at level $n$ and $e_{n+1} \quad(0, \ldots, 0,1)^{\mathrm{T}} \in \mathbb{R}^{n+1}$. Considering relation (2.24) in (2.23) leads us to

$$
x(\mathbf{A})_{n+1}(\mathbf{P})_{n} \quad\left[\left(\mathbf{J}_{Q}\right)_{n+1}(\mathbf{A})_{n+1}+e_{n+1}\left(s_{n+1} e_{n+1}^{T}+t_{n+1} e_{n}^{T}+r_{n+1} e_{n}^{T} 1\right)\right](\mathbf{P})_{n}+P_{n+1} e_{n+1},
$$

where $e_{n} \quad(0, \ldots, 0,1,0)^{\mathrm{T}}$ and $e_{n} 1 \quad(0, \ldots, 0,1,0,0)^{\mathrm{T}}$ are elements of $\mathbb{R}^{n+1}$. Replacing (2.22) in the last equality gives

$$
(\mathbf{A})_{n+1}\left(\mathbf{J}_{P}\right)_{n+1} \quad\left(\mathbf{J}_{Q}\right)_{n+1}(\mathbf{A})_{n+1}+(\mathbf{A})_{n+1} e_{n+1}\left(s_{n+1} e_{n+1}^{T}+t_{n+1} e_{n}^{T}+r_{n+1} e_{n}^{T} \quad 1\right) .
$$

Thus, we find the truncated form of $\mathbf{J}_{Q}$ by a rank-one perturbation of the corresponding truncated form of $\mathbf{J}_{P}$ with

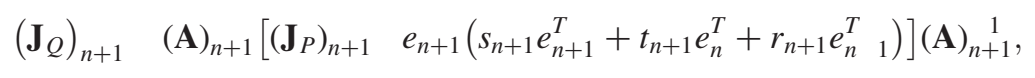

that is to say, $\left(\mathbf{J}_{Q}\right)_{n+1}$ is a rank-one perturbation of the matrix $\left(\mathbf{J}_{P}\right)_{n+1}$. 


\section{Special cases}

In this section, we will discuss some special cases of relation (1.3).

In [14], the author pointed out that Bernstein Szegö polynomials can be represented as a linear combination of Chebyshev polynomials with constant coefficients, i.e.

$$
Q_{n, i}(x) \quad P_{n, i}(x)+a_{1, i} P_{n} \quad 1, i(x)+\cdots+a_{k, i} P_{n} \quad k, i(x), \quad n>k,
$$

where $\left\{P_{n, i}\right\}_{n \geq 0}$ is the sequence of Chebyshev polynomials of $i$ th kind $(i \quad 1,2,3,4)$ and $a_{k, i} \neq 0$. Then, each kind of Bernstein Szegö polynomials $\left\{Q_{n, i}\right\}_{n \geq 0}(i \quad 1,2,3,4)$ is a sequence of orthogonal polynomials with respect to weight functions $\omega_{i}(x)$ if and only if

$$
\omega_{i}(x) \quad \frac{\mu_{i}(x)}{\sigma_{k}(x)}, \quad(i \quad 1,2,3,4),
$$

$\mu_{i}(x)$ is the Chebyshev weight function of each kind $(i \quad 1,2,3,4)$ and $\sigma_{k}(x)$ is a positive polynomial of degree $k$ on $(1,1)$.

In relation (1.3), let us assume that the coefficients are constant

$$
Q_{n}(x) \quad P_{n}(x)+h_{1} P_{n} \quad 1(x)+h_{2} P_{n} \quad 2(x)+h_{3} P_{n} \quad 3(x), \quad n \geq 4,
$$

where $h_{1}, h_{2}, h_{3} \in \mathbb{R}$ and $h_{3} \neq 0$. This means that the sequence $\left\{Q_{n}\right\}_{n \geq 0}$ is quasiorthogonal of order 3 with respect to the linear functional $u$ (see [6]). Under this hypothesis, from Theorem 2.1, we obtain necessary and sufficient conditions for the orthogonality of $\left\{Q_{n}\right\}_{n \geq 0}$ given by (3.1). Indeed,

$$
\tilde{\gamma}_{n} \quad \gamma_{n}+h_{1}\left(\begin{array}{lll}
\beta_{n} & 1 & \beta_{n}
\end{array}\right) \neq 0, \quad n \geq 4,
$$

and for $n \geq 5$

$$
\begin{aligned}
& h_{2}\left(\begin{array}{lll}
\beta_{n} & \beta_{n} & 2
\end{array}\right) \quad h_{1}\left(\begin{array}{llll}
\gamma_{n} & 1 & \gamma_{n} & 3
\end{array}\right) \text {, } \\
& h_{3}\left(\begin{array}{lll}
\beta_{n} & \beta_{n} & 3
\end{array}\right) \quad h_{2}\left(\begin{array}{llll}
\gamma_{n} & 2 & \gamma_{n} & 3
\end{array}\right) \text {, }
\end{aligned}
$$

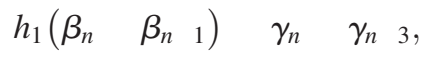

where the recurrence coefficients for the SMOP $\left\{Q_{n}\right\}_{n \geq 0}$ are

$$
\tilde{\gamma}_{n} \quad \gamma_{n}+h_{1}\left(\begin{array}{lll}
\beta_{n} & 1 & \beta_{n}
\end{array}\right), \quad n \geq 4, \quad \tilde{\beta}_{n} \quad \beta_{n}, \quad n \geq 4 .
$$

These orthogonality conditions are also obtained in [4]. Now, our aim is to define all SMOP $\left\{P_{n}\right\}_{n \geq 0}$ such that $\left\{Q_{n}\right\}_{n \geq 0}$ given by (3.1) is also orthogonal.

(i) Case $h_{1} \quad 0$.

In this case, we will analyse two subcases.

(a) $h_{2} \quad 0$.

In such a situation, it is clear from (3.2) (3.4) that for $n \geq 5$

$$
\beta_{n} \quad \beta_{n} 3, \quad \gamma_{n} \quad \gamma_{n} 3 .
$$

Hence, $\left\{P_{n}\right\}_{n \geq 0}$ is a three-periodic SMOP up to the initial conditions $\beta_{0}, \beta_{1}, \gamma_{1}$. Then, $\left\{Q_{n}\right\}_{n \geq 0}$ is also a three-periodic SMOP with the recurrence coefficients

$$
\tilde{\gamma}_{n} \quad \gamma_{n}, \quad \tilde{\beta}_{n} \quad \beta_{n}, \quad n \geq 4 .
$$


Sequences of monic orthogonal polynomials with recurrence relations with periodic coefficients were firstly studied by Geronimus (see [13] and [12]) as well as in [21]. Such SMOP can be expressed in terms of a polynomial mapping on the Chebyshev polynomials of the second kind (suitably shifted and rescaled) as was proved in Theorem 5.1 [8]. For a general theory of orthogonality and polynomial mappings see [11].

(b) $h_{2} \neq 0$.

From (3.2) (3.4), we get for $n \geq 5$

$$
\begin{aligned}
& \beta_{n} \quad \beta_{n} 2, \\
& \begin{array}{lll}
\gamma_{n} & \gamma_{n} & 3
\end{array}, \\
& h_{3}\left(\begin{array}{lll}
\beta_{n} & \beta_{n} & 3
\end{array}\right) \quad h_{2}\left(\begin{array}{llll}
\gamma_{n} & 2 & \gamma_{n} & 3
\end{array}\right) .
\end{aligned}
$$

By combining (3.5) and (3.7), we obtain

$$
h_{3}\left(\begin{array}{llll}
\beta_{n} & 2 & \beta_{n} & 3
\end{array}\right) \quad h_{2}\left(\begin{array}{llll}
\gamma_{n} & 2 & \gamma_{n} & 3
\end{array}\right), \quad n \geq 5,
$$

that is to say,

$$
h_{2} \gamma_{n} \quad h_{3} \beta_{n}+C, \quad n \geq 3,
$$

where $C \quad h_{2} \gamma_{2} \quad h_{3} \beta_{2}$ is a fixed constant. According to (3.5) and the last equalities, we have

$$
\gamma_{n} \quad \gamma_{n_{2}}, \quad n \geq 5
$$

and together with (3.6)

$$
\gamma_{n} \quad \gamma_{2}, \quad n \geq 2
$$

Furthermore, from (3.8)

$$
\beta_{n_{2}} \beta_{n_{3}}, \quad n \geq 5 \text {, i.e., } \beta_{n} \quad \beta_{2}, \quad n \geq 2,
$$

with $\beta_{2} \quad\left(1 / h_{3}\right)\left(h_{2} \gamma_{2} \quad C\right)$. Then, $\left\{P_{n}\right\}_{n \geq 0}$ is an SMOP with constant recurrence coefficients and $\beta_{2} \quad\left(1 / h_{3}\right)\left(h_{2} \gamma_{2} \quad C\right)$ but $\beta_{0}, \beta_{1}, \gamma_{1}$ are free parameters. These yield a perturbation in the initial conditions of Chebyshev polynomials of second kind, together with a shift in the variable. On the other hand, $\left\{Q_{n}\right\}_{n \geq 0}$ is also an SMOP with constant recurrence coefficients

$$
\tilde{\gamma}_{n} \quad \gamma_{n} \quad \gamma_{2}, \quad \tilde{\beta}_{n} \quad \beta_{n} \quad \beta_{2}, \quad n \geq 4 .
$$

(ii) Case $h_{1} \neq 0$.

In this case, the following two subcases will appear.
(a) $h_{2} \quad 0$. 
The conditions (3.2) (3.4) become for $n \geq 5$

$$
\begin{array}{cccc}
\gamma_{n} & 1 & \gamma_{n} & \\
& & \\
\beta_{n} & \beta_{n} & 3, & \\
\beta_{n} & \beta_{n} & 1 & \frac{1}{h_{1}}\left(\begin{array}{lll}
\gamma_{n} & \gamma_{n} & 3
\end{array}\right) .
\end{array}
$$

By using (3.9) (3.11), we find

$$
\beta_{n} \quad \beta_{n} 1 \frac{1}{h_{1}}\left(\begin{array}{lll}
\gamma_{n} & \gamma_{n} 1
\end{array}\right), \quad n \geq 5 \text {, i.e., } \quad \beta_{n} \frac{1}{h_{1}} \gamma_{n}+C^{*}, \quad n \geq 5,
$$

where $C^{*} \quad \beta_{4} \quad\left(1 / h_{1}\right) \gamma_{4}$ is a fixed constant. Similar computations lead us to

$$
\beta_{n} \quad \beta_{2}, \quad n \geq 2, \quad \gamma_{n} \quad \gamma_{2}, \quad n \geq 2,
$$

with $\beta_{2} \quad\left(1 / h_{1}\right) \gamma_{2}+C^{*}$. Thus, $\left\{P_{n}\right\}_{n \geq 0}$ is an SMOP with constant recurrence coefficients defined by the above equalities and $\beta_{2} \quad\left(1 / h_{1}\right) \gamma_{2}+C^{*}$ while $\beta_{0}, \beta_{1}, \gamma_{1}$ are free parameters. Then, we have a similar situation to that analysed in (b) of (i). Furthermore, the recurrence coefficients of the SMOP $\left\{Q_{n}\right\}_{n \geq 0}$ are

$$
\begin{array}{ll}
\tilde{\gamma}_{n} & \gamma_{n}+h_{1}\left(\begin{array}{lll}
\beta_{n} & 1 & \beta_{n}
\end{array}\right) \quad \gamma_{2}, \quad n \geq 4, \\
\tilde{\beta}_{n} & \beta_{n} \quad \beta_{2}, \quad n \geq 4 .
\end{array}
$$

(b) $h_{2} \neq 0$.

Taking into account (3.2) (3.4), we deduce that $\beta_{n}$ and $\gamma_{n}$ satisfy the following difference equation

$$
y_{n}+\left(\begin{array}{ll}
1 & \frac{h_{2}^{2}}{h_{1} h_{3}}
\end{array}\right) y_{n} \quad 1 \quad\left(\begin{array}{ll}
1 & \frac{h_{2}^{2}}{h_{1} h_{3}}
\end{array}\right) y_{n} \quad 3 \quad y_{n} \quad 4 \quad 0, \quad n \geq 6 .
$$

The characteristic equation of the above difference equation is

$$
\left(\begin{array}{ll}
\lambda^{2} & 1
\end{array}\right)\left[\lambda^{2}+\left(\begin{array}{ll}
1 & \frac{h_{2}^{2}}{h_{1} h_{3}}
\end{array}\right) \lambda+1\right] \quad 0 .
$$

The solution of the characteristic equation (3.13) depends on the behaviour of the constant $h_{2}^{2} / h_{1} h_{3}$ (see [9]).

(b $\mathrm{b}_{1}$ ) If $h_{2}^{2} \quad h_{1} h_{3}$, then, from (3.13), $\lambda \quad 1$ is a zero with multiplicity 3 . Thus,

$$
\begin{aligned}
& \beta_{n} \quad f_{11}+\left[f_{12}+f_{13} n+f_{14} n^{2}\right](1)^{n}, \quad n \geq 2, \\
& \gamma_{n} \quad g_{11}+\left[g_{12}+g_{13} n+g_{14} n^{2}\right](\quad 1)^{n}, \quad n \geq 2 \text {. }
\end{aligned}
$$

Moreover, $f_{1 j}$ and $g_{1 j}$ are related according to (3.2) (3.4).

$\left(\mathrm{b}_{2}\right)$ If $h_{2}^{2} \quad 3 h_{1} h_{3}$, then, from (3.13), $\lambda \quad 1$ is a zero with multiplicity 3 . Therefore,

$$
\begin{array}{lll}
\beta_{n} & f_{21}+f_{22} n+f_{23} n^{2}+f_{24}(1)^{n}, & n \geq 2, \\
\gamma_{n} & g_{21}+g_{22} n+g_{23} n^{2}+g_{24}(1)^{n}, & n \geq 2 .
\end{array}
$$


$\left(\mathrm{b}_{3}\right)$ If $\left(h_{2}^{2} / h_{1} h_{3}\right) \in \mathbb{R} \backslash[1,3]$, then from (3.13)

$$
\begin{array}{lll}
\beta_{n} & f_{31}+f_{32}(1)^{n}+f_{33} \lambda^{n}+f_{34} \lambda^{n}, & n \geq 2, \\
\gamma_{n} & g_{31}+g_{32}(1)^{n}+g_{33} \lambda^{n}+g_{34} \lambda & n
\end{array}
$$

Here, $\lambda$ is the unique solution of equation (3.13) such that $\lambda \in(1,1)$.

$\left(\mathrm{b}_{4}\right)$ If $\left(h_{2}^{2} / h_{1} h_{3}\right) \in(1,3)$, then from (3.13)

$$
\begin{array}{lll}
\beta_{n} & f_{41}+f_{42}(1)^{n}+f_{43} \mathrm{e}^{i n \theta}+f_{44} \mathrm{e}^{i n \theta}, & n \geq 2, \\
\gamma_{n} & g_{41}+g_{42}(1)^{n}+g_{43} \mathrm{e}^{i n \theta}+g_{44} \mathrm{e}^{i n \theta}, & n \geq 2,
\end{array}
$$

where $\lambda \quad \mathrm{e}^{i \theta}$ is the unique solution of equation (3.13) with $\theta \in(0, \pi)$.

Remark 3.1. It is worthy to notice that each $\left\{P_{n}\right\}_{n \geq 0}$ given by the recurrence coefficients $\beta_{n}$ and $\gamma_{n}$ explicitly obtained in $\left(\mathrm{b}_{1}\right) \quad\left(\mathrm{b}_{4}\right)$ is a SMOP but $\beta_{0}, \beta_{1}, \gamma_{1}$ are free parameters.

Remark 3.2. In each situation $\left(b_{1}\right) \quad\left(b_{4}\right)$, the recurrence coefficients of the SMOP $\left\{Q_{n}\right\}_{n \geq 0}$ are

$$
\tilde{\gamma}_{n} \quad \gamma_{n}+h_{1}\left(\begin{array}{lll}
\beta_{n} & 1 & \beta_{n}
\end{array}\right), \quad n \geq 4, \quad \tilde{\beta}_{n} \quad \beta_{n}, \quad n \geq 4,
$$

with the condition $\gamma_{n}+h_{1}\left(\begin{array}{lll}\beta_{n} & 1 & \beta_{n}\end{array}\right) \neq 0, n \geq 4$, where $\beta_{n}$ and $\gamma_{n}$ are obtained in $\left(b_{1}\right) \quad\left(b_{4}\right)$.

Remark 3.3. The comparison with the results given in [4,2] shows that the periodic character of the coefficients of the three-term recurrence relation pointed out in (i) and (ii) (a) can be expected but the coefficients of the three-term recurrence relation obtained in (ii) (b) are apparently unexpected. An interesting problem is the analysis of the integral representation of the linear functionals associated with the SMOP such that the coefficients of the three-term recurrence relation are described in (ii) (b). As far as we know they are not yet studied in the literature of orthogonal polynomials (see [6] and [15]). Notice that those described in (i) and (ii) (a) are very well known. This constitutes an added value of the problem analysed in our contribution.

Remark 3.4. If relation (3.1) holds for $n \geq 3$, then we also have

$$
\begin{array}{llll}
\tilde{\gamma}_{3} & \gamma_{3}+h_{1}\left(\beta_{2}\right. & \left.\beta_{3}\right), \quad \tilde{\beta}_{3} & \beta_{3} .
\end{array}
$$

Notice that in order to have orthogonality, the condition $\tilde{\gamma}_{3} \neq 0$ must hold. On the other hand,

$$
s_{2} \frac{1}{\tilde{\gamma}_{3}}\left(h_{1} \gamma_{2}+h_{2}\left(\beta_{1} \quad \beta_{3}\right)\right)
$$

as well as

$$
t_{2} \quad \frac{1}{\tilde{\gamma}_{3}}\left(h_{2} \gamma_{1}+h_{3}\left(\beta_{0} \quad \beta_{3}\right)\right) .
$$

Thus, we get the conditions about these two parameters that give the explicit expression for $Q_{2}(x)$. On the other hand, from (2.5) and (2.6)

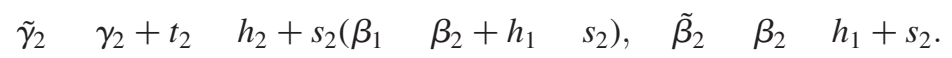


Notice that in order to have orthogonality, the condition $\tilde{\gamma}_{2} \neq 0$ must be satisfied. On the other hand,

$$
s_{1} \quad \frac{1}{\tilde{\gamma}_{2}}\left(s_{2} \gamma_{1} \quad h_{3}+t_{2}\left(\beta_{0} \quad \beta_{2}+h_{1} \quad s_{2}\right)\right)
$$

Finally,

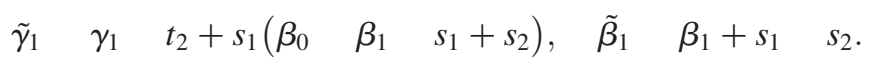

Notice that in order to have orthogonality, the condition $\tilde{\gamma}_{1} \neq 0$ must be satisfied. As an immediate consequence, one has $\tilde{\beta}_{0} \quad \beta_{0} \quad s_{1}$. Thus, all the coefficients of the three-term recurrence relation for the sequence $\left\{Q_{n}\right\}_{n \geq 0}$ are completely determined.

As an application of the above results, we will focus our attention in the case when the sequence $\left\{P_{n}\right\}_{n \geq 0}$ is symmetric, i.e. $\beta_{n} \quad 0$ for every $n \geq 0$. The above conditions yield

$$
\tilde{\gamma}_{n} \quad \gamma_{n}, \quad n \geq 3, \quad \tilde{\beta}_{n} \quad \beta_{n}, \quad n \geq 3 .
$$

Furthermore,

$$
s_{2} \quad h_{1} \frac{\gamma_{2}}{\gamma_{3}}
$$

as well as

$$
t_{2} \quad h_{2} \frac{\gamma_{1}}{\gamma_{3}}
$$

On the other hand,

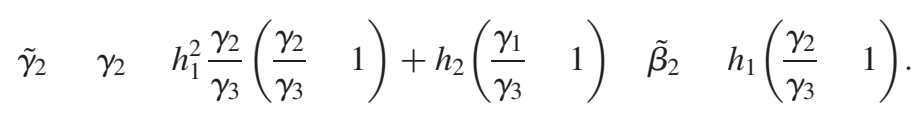

Notice that if $\left\{P_{n}\right\}_{n \geq 0}$ is the sequence of monic Chebyshev polynomials of second kind, the above conditions become

$$
\tilde{\gamma}_{n} \frac{1}{4}, \quad \tilde{\beta}_{n} \quad 0, \quad n \geq 2, \quad s_{2} \quad h_{1}, t_{2} \quad h_{2},
$$

as well as

$$
s_{1} \quad h_{1} \quad 4 h_{3} .
$$

In this case,

$$
\tilde{\gamma}_{1} \frac{1}{4} \quad h_{2}+4 h_{3}\left(h_{1} \quad 4 h_{3}\right), \quad \tilde{\beta}_{1} \quad 4 h_{3}, \quad \tilde{\beta}_{0} \quad 4 h_{3} \quad h_{1} .
$$

In order to have orthogonality, one can fix any $h_{1}$ and $h_{3} \neq 0$ in such a way $h_{2} \neq(1 / 4)+4 h_{3}\left(4 h_{3} \quad h_{1}\right)$.

If $\left\{P_{n}\right\}_{n \geq 0}$ is the sequence of monic Hermite polynomials, the above conditions become

$$
\tilde{\gamma}_{n} \frac{n}{2}, \quad \tilde{\beta}_{n} \quad 0, \quad n \geq 3, s_{2} \quad \frac{2 h_{1}}{3}, t_{2} \quad \frac{h_{2}}{3} .
$$

Thus

$$
\tilde{\gamma}_{2} \quad 1 \quad \frac{2 h_{2}}{3}+\frac{2 h_{1}^{2}}{9}, \tilde{\beta}_{2} \quad \frac{h_{1}}{3}
$$


The orthogonality condition means that $h_{2} \neq\left(2 h_{1}^{2}+9\right) / 6$. On the other hand,

$$
s_{1} \frac{1}{\tilde{\gamma}_{2}}\left(\begin{array}{lll}
\frac{h_{1}}{3} & h_{3} & \frac{h_{1} h_{2}}{9}
\end{array}\right) .
$$

Finally,

$$
\tilde{\gamma}_{1} \frac{1}{2} \frac{h_{2}}{3}+s_{1}\left(\frac{2 h_{1}}{3} \quad s_{1}\right), \quad \tilde{\beta}_{1} \quad s_{1} \quad \frac{2 h_{1}}{3}, \quad \tilde{\beta}_{0} \quad s_{1} .
$$

Thus, the orthogonality condition yields another constraint about the possible choices of $h_{3}$. This Hermite case shows that, for a fixed $h_{1}$, one can choose $h_{2}$ and $h_{3} \neq 0$ in order to have orthogonality if and only if one has the above constraints.

\section{Acknowledgements}

First of all, the authors like to thank the referees for their careful revision of the manuscript. Their comments and suggestions have substantially improved the presentation of the manuscript. The work of the first author (FM) has been supported by Ministerio de Economía y Competitividad of Spain, grant MTM 201236732 C03 01. This paper was completed during a stay of the second author (SV) in the Department of Mathematics of Universidad Carlos III de Madrid in the spring semester of the academic year 2012 2013. He acknowledges the kind reception there.

\section{Note}

1. Email: svarma@science.ankara.edu.tr

\section{References}

[1] M. Alfaro, A. Peña, J. Petronilho, and M.L. Rezola, Orthogonal polynomials generated by a linear structure relation: Inverse problem, J. Math. Anal. Appl. 401(1) (2013), pp. 182197.

[2] M. Alfaro, A. Peña, M.L. Rezola, and F. Marcellán, Orthogonal polynomials associated with an inverse quadratic spectral transform, Comput. Math. Appl. 61 (2011), pp. 888900.

[3] M. Alfaro, F. Marcellán, A. Peña, and M.L. Rezola, On linearly related orthogonal polynomials and their functionals, J. Math. Anal. Appl. 287 (2003), pp. 307319.

[4] M. Alfaro, F. Marcellán, A. Peña, and M.L. Rezola, When do linear combinations of orthogonal polynomials yield new sequences of orthogonal polynomials? J. Comput. Appl. Math. 233 (2010), pp. 14461452.

[5] A. Branquinho and F. Marcellán, Generating new classes of orthogonal polynomials, Int. J. Math. Math. Sci. 19 (1996), pp. 643656.

[6] T.S. Chihara, An Introduction to Orthogonal Polynomials, Gordon and Breach, New York, NY, 1978.

[7] T.S. Chihara, On co recursive orthogonal polynomials, Proc. Amer. Math. Soc. 8 (1957), pp. 899905.

[8] M.N. de Jesus and J. Petronilho, On orthogonal polynomials obtained via polynomial mappings, J. Approx. Theory 162 (2010), pp. 22432277.

[9] S. Elaydi, An Introduction to Difference Equations, Springer, New York, NY, 2005.

[10] W. Gautschi, Orthogonal Polynomials: Computation and Approximation, Numerical Mathematics and Scientific Computation. Oxford University Press, New York, NY, 2004.

[11] J.S. Geronimo and W. Van Assche, Orthogonal polynomials on several intervals via a polynomial mapping, Trans. Amer. Math. Soc. 308 (1988), pp. 559581.

[12] Ya. L. Geronimus, On some finite difference equations and corresponding systems of orthogonal polynomials, Mem. Math. Sect. Fac. Phys. Kharkov State Univ. Kharkov Math. Soc 25 (1975), pp. 81100.

[13] Ya. L. Geronimus, Sur quelques équations aux différences finies et les systèmes correspondants des polynômes orthogonaux. C.R. (Dokl.), Acad. Sci. URSS 29 (1940), pp. 536538. 
[14] Z. Grinshpun, Special linear combinations of orthogonal polynomials, J. Math. Anal. Appl. 299 (1) (2004), pp. 118.

[15] R. Koekoek, P.A. Lesky, and R.F. Swarttouw, Hypergeometric orthogonal polynomials and their q analogues. With a foreword by Tom H. Koornwinder, Springer Monographs in Mathematics. Springer Verlag, Berlin, 2010.

[16] F. Marcellán and J. Petronilho, Orthogonal polynomials and coherent pairs: The classical case, Indag. Math. (NS) 6 (1995), pp. 287307.

[17] P. Maroni and I. Nicolau, On the inverse problem of the product of a form by a polynomial: The cubic case, Appl. Numer. Math. 45(4) (2003), pp. 419451.

[18] P. Maroni, Semi classical character and finite type relations between polynomial sequences, Appl. Numer. Math. 31(3) (1999), pp. 295330.

[19] P. Maroni, Sur la suite de polynômes orthogonaux associée à la forme $u=\delta_{c}+\lambda(x \quad c){ }^{1} L$, Period. Math. Hungar. 21(3) (1990), pp. 223248.

[20] P. Maroni, Une théorie algébrique des polynômes orthogonaux. Application aux polynômes orthogonaux semi classiques, in Orthogonal Polynomials and their Applications, IMACS Ann. Comput. Appl. Math. C. Brezinski, L. Gori, and A. Ronveaux, eds., Vol. 9, Baltzer, Basel, 1991, pp. 95130.

[21] F. Peherstorfer, On Bernstein Szegó orthogonal polynomials on several intervals. II. Orthogonal polynomials with periodic recurrence coefficients, J. Approx. Theory 64 (1991), pp. 123161.

[22] J. Petronilho, On the linear functionals associated to linearly related sequences of orthogonal polynomials, J. Math. Anal. Appl. 315 (2006), pp. 379393.

[23] V.B. Uvarov, The connection between systems of polynomials that are orthogonal with respect to different distribution functions, Comput. Math. Math. Phys. 9 (1969), pp. 2536.

[24] A. Zhedanov, Rational spectral transformations and orthogonal polynomials, J. Comput. Appl. Math. 85 (1997), pp. 6786. 